\title{
Design and Analysis of Satellite Based Automatic Identification System in VHF Band for Maritime Applications
}

\author{
Ganesh Narasimhan ", Rajendran Velayutham \\ Department of Electronics and Communication Engineering, VELS University, Chennai, India \\ Email address: \\ Enganesh50@gmail.com (G. Narasimhan) \\ ${ }^{*}$ Corresponding author
}

\section{To cite this article:}

Ganesh Narasimhan, Rajendran Velayutham. Design and Analysis of Satellite Based Automatic Identification System in VHF Band for Maritime Applications. International Journal of Wireless Communications and Mobile Computing. Vol. 7, No. 2, 2019, pp. 32-37.

doi: $10.11648 /$ j.wcmc.20190702.11

Received: February 1, 2019; Accepted: September 2, 2019; Published: September 17, 2019

\begin{abstract}
This paper is introducing analyses of new design of the Automatic Identification System (AIS) ships antenna that will serve for both VHF-band Radio and Satellite tracking and identification system for maritime applications. The current AIS network is an automatic tracking system used by ships of Vessel Traffic Service (VTS) for identification and location of vessels by electronically exchanging data between nearby ships and on-shore base station. In the AIS system for the electronic data exchange, the Very High Frequency (VHF) range is used. Utilizing of the VHF range makes the AIS as short-range communication, identification and collision avoidance system between AIS equipped ships and base station is possible in the antennas Line-of-Sight (LOS). In order to widen the range of the AIS from short range up to long or global range should be used AIS communication via a satellite. In order to provide communication via satellite as well should be designed multifunctional antenna for both Radio AIS (R-AIS) and Satellite AIS (S-AIS) communication antenna. The analyses and design of a new multifunctional antenna, calculation and development, including implementation and testing of helical VHF antenna for R-AIS and S-AIS equipment are discussed.
\end{abstract}

Keywords: Very High Frequency, Maritime, Data Link and Line of Sight

\section{Introduction}

The VHF Radio AIS (R-AIS) or Radio VHF Data Link (RVDL) is a most attractive system at present for short range tracking and detecting of ships in coastal navigation. Because of limited R-AIS range recently is proposed Satellite AIS (SAIS) for global coverage via Inmarsat, Iridium or Orbcomm satellite constellation. Regulation 19 of the SOLAS Chapter $\mathrm{V}$ provided requirements for shipborne navigational systems and equipment and sets out navigational equipment to be carried onboard ships, according to ship type. In 2000, IMO adopted a new requirement, as part of a revised new chapter $\mathrm{V}$, for all ships to carry AIS transponders capable of providing information about the ship to other ships and to coastal authorities automatically. The R-AIS is a maritime surveillance system using the VHF band to exchange information between ships and shore stations, including positions, identification, course and speed, which network is shown in Figure 1. It mainly aims at avoiding collisions between ships. The link budgets allow receiving transmitted AIS signals from space, and consequently a global maritime surveillance can be considered. However, some challenges arise, especially message collisions due to the use of a Self Organized Time Division Multiple Access (SOTDMA) protocol (not designed for satellite detecting). Thus, advanced signal processing for separation of received signals is needed.

According to IMO regulations by 31 December 2004 each oceangoing vessel has to install AIS transponder devices onboard, which automatically broadcast regularly to the coast station ships name, call sign and navigation data. This data is programmed when the equipment is installing onboard ships and also all this information is transmitted regularly. The signals are received by R-AIS transponders fitted on other 
ships or on land based systems, such as Vessel Monitoring System (VMS). The received information can be displayed on a screen or chart plotter, showing the other nearby vessel's positions in much the same manner as a radar display [1] and also the information can be shown in display graph as in [2]. IMO [3] described about this display of data in their manual. Den Hang [9] and Ensu [10] explained Marine AIS and Implications. C. Lihu gave details design of AIS using Density distribution of Vessels. [11]. L. Li described data based decision model of AIS and talks about risk over sea. [12]. IV Kartika etal enumerates the AIS Placement using genetic algorithm. [13]. The erection and positioning of Automatic Identification system (AIS) is explained in detail by Y J Wu etal in [14]. Finally S. Huwai and S. Zhang [15] described the AIS Signal using Sparse Representation.

\section{Analysis}

Classical dipole antenna for the VHF-band has quite simple construction suitable for many applications and in particular for radio and satellite AIS systems. The conventional dipole antenna has some disadvantages for AIS usage. The main aspects of VHF radio antenna analyzes concerning to VHF AIS are: Polarization, which is particular the same for VHF AIS; Radiation pattern of radio VHF antenna and antenna gain; Severe influence of different and extreme weather conditions on communication between ground and mobile AIS antenna units with different VHF radiation pattern and various antenna gains; and Ability of ship R-AIS antenna to communicate with VHF S-AIS satellite.

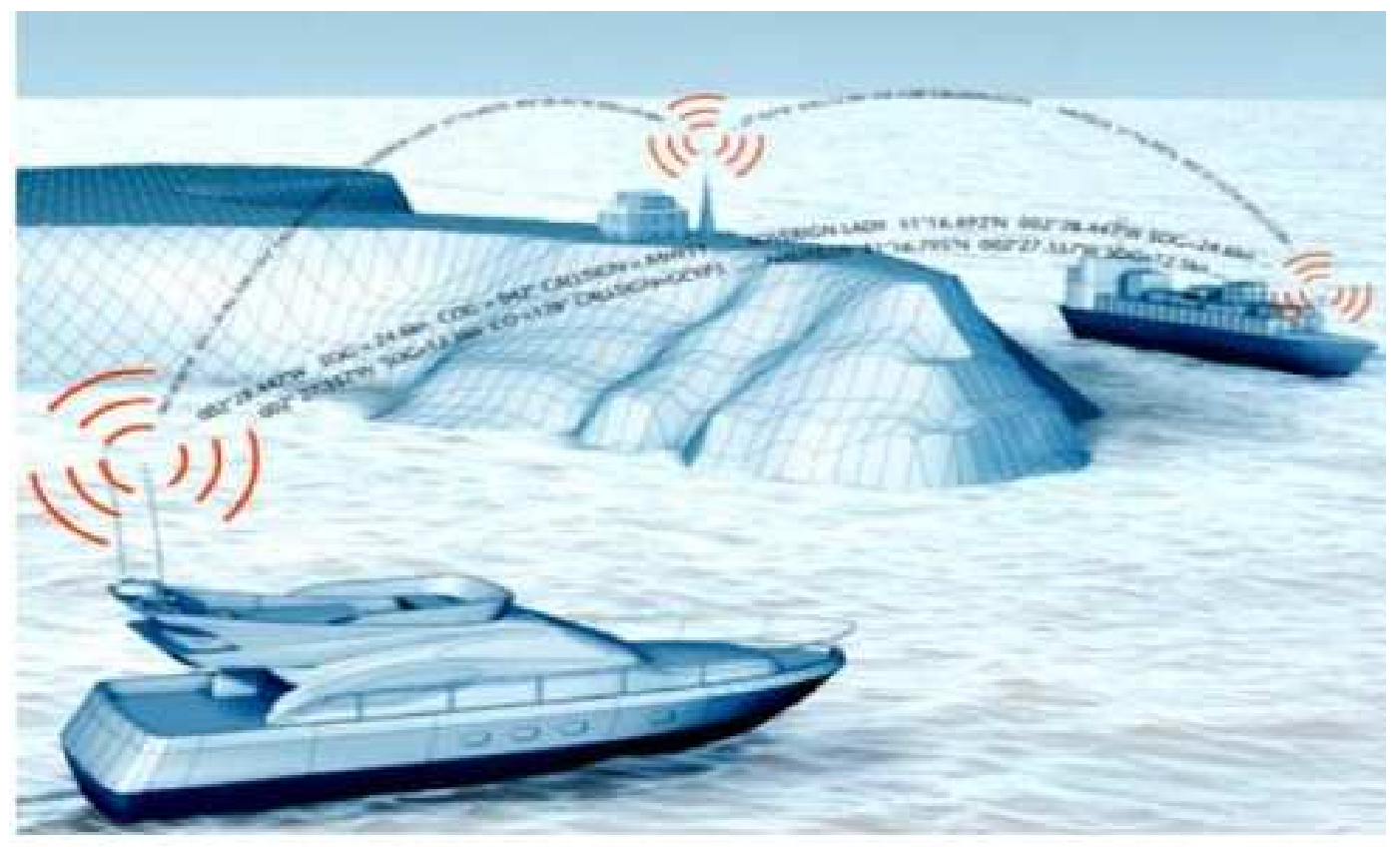

Figure 1. Maritime AIS network - courtesy of brochure [3].

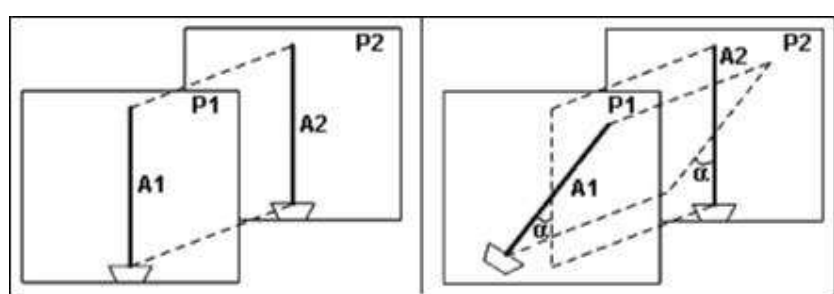

Figure 2. Inclination of AIS dipole Antenna [4].

Concerns vertical (used onboard ships) or horizontal polarization depending on antenna position in space. Both antennas are called a linear polarization, because component of radiated EM waves by such antenna type never changes own location in space. For both antennas, receiving and transmitting, simultaneous location for their installations becomes critical in working space onboard ships. In such a way, to provide reliable radio link will be necessary to employ linearly polarized AIS antennas, which reciprocal elements are placing strictly in parallel planes, otherwise ships radio communication becomes significantly not affordable. This inclination of dipole antenna process for simultaneous placing of linearly polarized antennas is shown in Figure 2 (Left and Right). Thus, the left side of figure shows inclination between antennas $\mathrm{A} 1$ and $\mathrm{A} 2 \alpha=00$ as a condition of the best communication case, and right side of figure shows inclination between antennas A1 and A2 $\alpha>00$ as a condition of worsening communication. In fact, the best communication of linearly polarized antenna is when simultaneous inclination between the antennas A1 and A2 in parallel planes $\mathrm{P} 1$ and $\mathrm{P} 2$ is equals to $\alpha=00$. This case is experiencing during good whether conditions, when pitching and rolling of a ship is not causing negative fluctuation to the antenna reception. In contrary, radio communication becomes worse when simultaneous inclination is at $0<\alpha<900$ and there is not radio communication at all when inclination between antennas $\mathrm{A} 1$ and $\mathrm{A} 2$ is equals to $\alpha=90^{\circ}$. The radiation 
pattern of antenna depends on antenna gain, so the higher antenna's gain, the more concentrates transmitting power or focuses radiating power. The dipole antennas with a high $\mathrm{dB}$ rating concentrates energy perpendicular to the antenna shaft in a field that is shaped like a disk or "donate". To provide mathematical analyze of dipole antenna radiation pattern, will be used well known Maxwell's equations and with equation antenna directivity. The equation for radiation intensity is radiated power per solid angle. The dipole antenna radiation pattern (case antenna gain is $3 \mathrm{~dB}$ ) is shown in Figure $3[1,2,4]$.

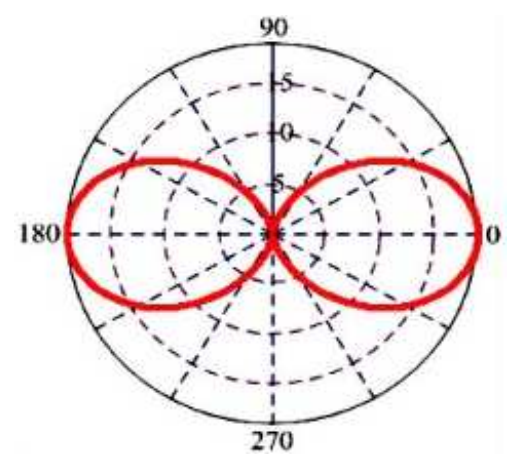

Figure 3. Dipole antenna radiation pattern.

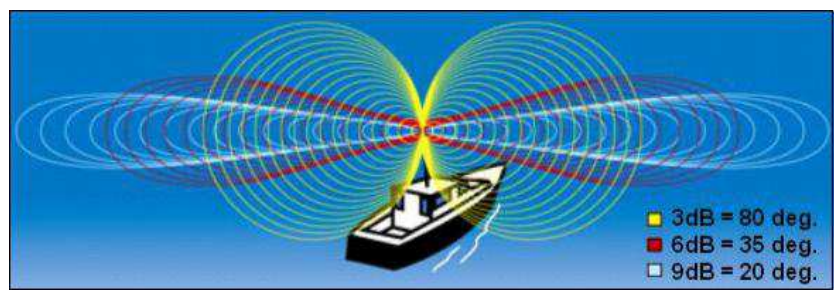

Figure 4. Dipole radiation pattern at different antenna gains [5].

In the far field radiation intensity is following:

$\operatorname{Prad}=\int_{\mathrm{o}}{ }^{2 \pi} \int_{\mathrm{O}}{ }^{\pi} \mathrm{U}(\theta, \varphi) \sin \theta \mathrm{d} \theta \mathrm{d} \varphi=\int_{\mathrm{O}}{ }^{2 \pi} \int_{\mathrm{O}}{ }^{\pi} \mathrm{U}(\theta, \varphi) \mathrm{d} \Omega$

where $d \Omega=\sin (\theta) d \theta d \varphi$ is differential solid angle.

The average radiation intensity is defined by dividing the radiation intensity (1) with the area of the unit sphere $(4 \pi)$, which relation gives:

$$
\operatorname{Uavg}=\int_{\mathrm{o}}{ }^{2 \pi} \int_{\mathrm{o}} \pi \mathrm{U}(\theta, \varphi) \mathrm{d} \Omega / 4 \pi=\operatorname{Prad} / 4 \pi
$$

The directivity antenna equation is the ratio of the radiation intensity (1) in a given direction from the antenna to the average radiation intensity (2) over all directions. Hence, formula for antenna directivity is as follows:

$$
\mathrm{D}(\theta, \varphi)=\mathrm{U}(\theta, \varphi) / \mathrm{Uavg}=4 \pi \mathrm{U}(\theta, \varphi) / \operatorname{Prad}
$$

Inserting the Prad expression into the directivity is:

$$
\mathrm{D}(\theta, \varphi)=4 \pi \mathrm{F}(\theta, \varphi) / \int_{\mathrm{o}} 2 \pi \int_{\mathrm{o}} \pi \mathrm{F}(\theta, \varphi) \sin (\theta) \mathrm{d} \theta \mathrm{d} \varphi
$$

where value $F(\theta, \varphi)=$ function of radiation pattern intensity. To plot radiation pattern, radiated pattern function of dipole antenna is needed, which mutual relation is presented by:

$$
\mathrm{F}(\theta)=0.64 \cos 2(\theta)
$$

Inserting formula (5) into (4) and with the help of software "MathCAD", dipole antenna radiation pattern diagram is plotted with a vertical cross-cut. In Figure 3 can be seen two lobes of radiation pattern known as main lobes of radiation pattern.

Radiation patterns of VHF dipole antenna for AIS system at different antenna gains (Yellow is $3 \mathrm{~dB}$, red is $6 \mathrm{~dB}$ and blue is 9 $\mathrm{dB}$ ) shown in Figure 4. Thus, the higher antenna gain the better radiating energy concentration is in space, which reduces the amount of radiated energy above or below the antenna perpendicular line toward antenna axis. In fact, should be considered communication of AIS equipped ships with the VHF high and low gain antenna providing 3 and $9 \mathrm{~dB}$, respectively. This consideration is vital to analyze and define necessary antenna gain for AIS. The radiation pattern of ship High Gain Antenna (HGA) concentrates radiating energy in narrow lobes along the horizontal line. The HGA array is suitable for longrange communication, owing to high concentration of radiation energy. However, this case is advantage only in normal navigation and weather/sea conditions without extremely pitching and rolling of a ship. In contrary, ships radio communication becomes significantly worse when the ship begins pitching or rolling due to agitated sea, hence the radiation pattern of VHF AIS antenna becomes inclined relatively to horizon line, what is shows in Figure 5 (Left). This figure shows a ship equipped with the VHF HGA. In case when VHF Low Gain Antenna (LGA) is used, main lobes of radiation pattern become significantly wider. The radiated energy by LGA quickly is spreading out, what significantly reduces communication range. But in case of very high sea agitation, when ship is pitching and rolling, the transmitting or receiving VHF antenna of nearby ships is still capable to receive or transmit signal as shown in Figure 5 (Right).

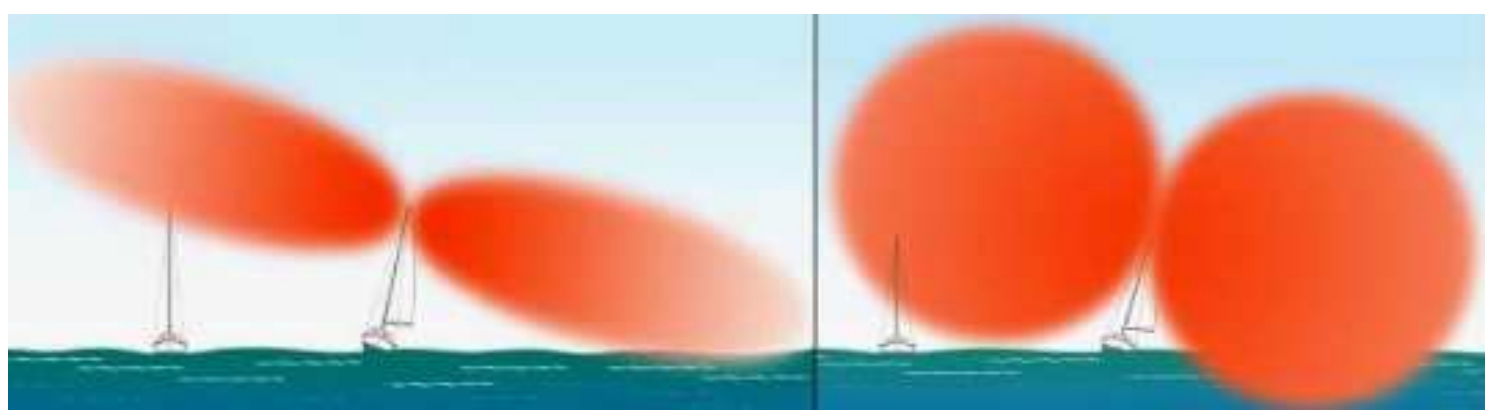

Figure 5. Ship equipped with HGA and Transmission with LGA [6]. 


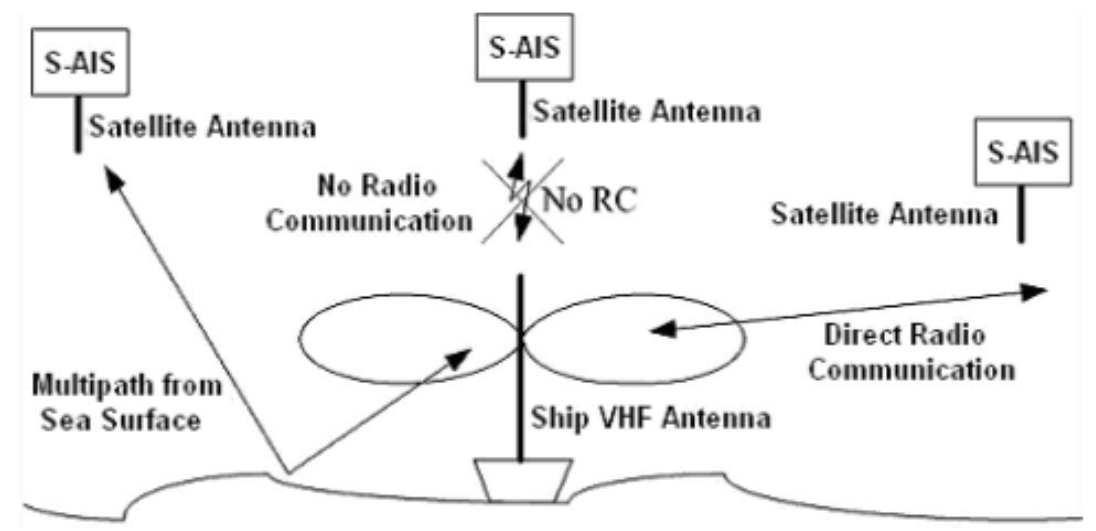

Figure 6. Graphic of Possible S-AIS via VHF Antenna [4].

It presents reliable satellite communication with standard AIS VHF antenna, which deserves special consideration. To provide link between AIS Orbcomm (S-AIS) satellite and AIS ship-borne terminal (R-AIS) should be used the same low gain VHF antenna as for R-AIS. According to the graphic analyses shown in Figure 6, satellite communication with the VHF antenna is possible in two following cases:

Radio wave reflection from sea water surface

Radiation pattern of ship-borne VHF antenna fully matches its radiation pattern with satellite's antenna in LOS. To improve radiation pattern, should be selected another type of maritime antenna for S-AIS, such as spiral or helical antenna, which radiates circular polarized signal.

\section{Calculation and Design of Helical Antenna}

In order to choose necessary construction of VHF helical antenna array should be considered environmental conditions where this antenna is going to be used. According to the current and latest research analyses, the most suitable antenna for S-AIS is Quadrifilar Helical Antenna (QHA). Consequently, it will be necessary precisely to select needed radiation pattern of QHA with open circuit, which can be plotted by the known radiation pattern formula for helical antenna:

$$
\mathrm{F}(\theta)=\cos (\theta) \sin (\mathrm{N} \psi / 2) / \sin (\psi / 2)
$$

where value $\mathrm{N}=$ number of turns for every given helix; and $\psi=\mathrm{kS} \cos (\theta)+\alpha=$ summarized variable determined by the type of circulation $\mathrm{k}$ and pitch angle of helix $\alpha$, which equation is:

$$
\alpha=\mathrm{s} / \pi \mathrm{d}
$$

where value $\mathrm{s}=$ space between turns of helix; and $\mathrm{d}=$ diameter of helix turns. Executing several simple conversions of equation provides the QHA formula and inserting converted equation (6) of QHA radiation pattern into equation (4) can be plotted chart by the "MathCAD" software.

The QHA radiation pattern is illustrated in Figure 7 (Left) and model of QHA resonant for R-AIS system is shown in Figure 7 (Right). Considering presented radiation pattern can be seen that radiation pattern has hemispherical shape, therefore, such radiation pattern is suitable for satellite communication and tracking (S-AIS) and including for (R-AIS) intercommunication between ships (ship-to-ship) due to the significant broad radiation pattern within horizontal plane.
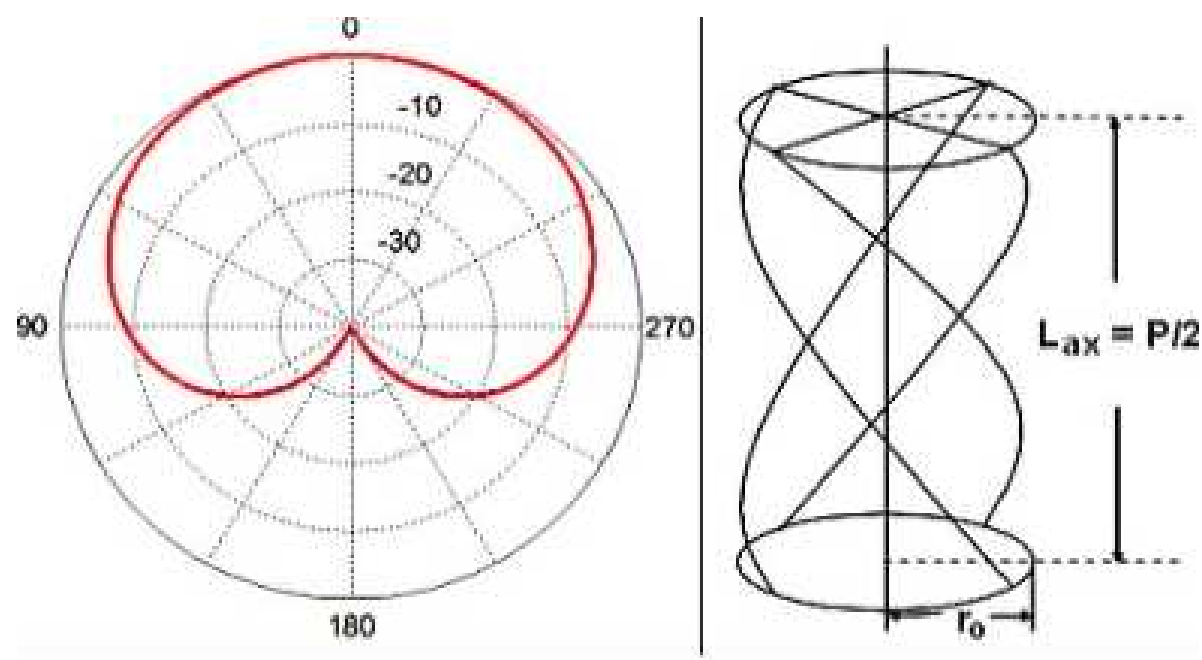

Figure 7. QHA Radiation Pattern and Model [4]. 

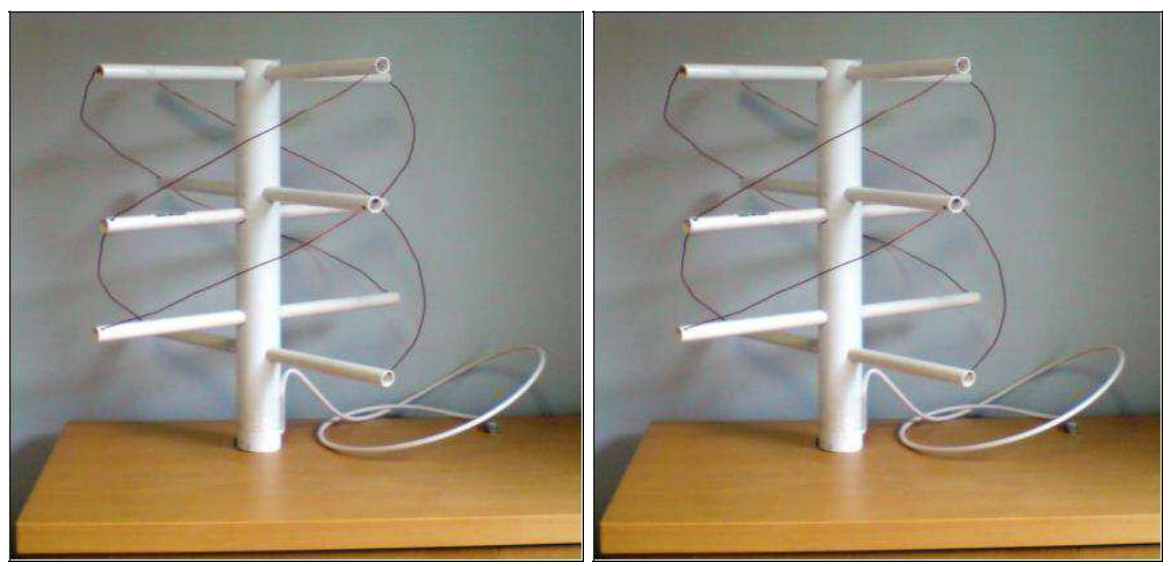

Figure 8. Experimental Sample of the QHA.

This resonant QHA antenna consists of four spiral arms separately fed by currents, which have equal amplitude and $90^{\circ}$ difference between two orthogonal. The resonant QHA antenna can be divided into two types, open and shorted circuit at non-feed end, which main parameters for implementation onboard ships are following: ro $=$ spiral radius; $\mathrm{P}=$ thread pitch; $\mathrm{N}=$ turns numbers; Lele $=$ helix length; and $\operatorname{Lax}=$ axial length. All these variables are included in equation:

$$
P=\sqrt{ }\left(1 / N^{2}(\text { Lele }- \text { Aro })^{2}-4 \pi^{2} \text { ro }^{2}\right.
$$

where values Lele $=(2 n+1) \lambda / 4$ (opened at non-feed); $n=$ $\mathrm{n} \lambda / 2$ (shorted at non-feed) both values are considered with $\mathrm{n}$ $=0,1,2, \ldots ; \mathrm{A}=1$ (if open at non-feed) and $\mathrm{A}=2$ (if shorted at non-feed). Also important aspect of the QHA antenna is auto-phase shifting structure that produces 900 differences through its antenna arms, so the length of two bifilar helices is different. The longer arm can produce a capacitive input impedance with negative 450 phase shift different to resonance, while the shorter gives resonant length that produces a inductive input impedance with positive 450 phase shift different to resonance. Therefore, two bifilar helices can produce 900 phase shift difference without any auxiliary structure used to produce phase shift. Also important aspect of the QHA antenna is auto-phase shifting structure that produces 900 differences through its antenna arms. The principle is that the length of two bifilar helices is different. This, one slightly longer than resonant length that can produce a capacitive input impedance with negative 450 phase shift different to resonance; another arm is slightly shorter than resonant length that produces a inductive input impedance with positive 450 phase shift different to resonance. Therefore, two bifilar helices can produce 900 phase shift difference without any auxiliary structure used to produce phase shift.

In addition, main parameter of the QHA antenna that determines size depends on central operation frequency, so R-AIS system uses two radio channels at frequencies of 161.975 and $162.025 \mathrm{MHz}$ and with spectrum width at 25 $\mathrm{kHz}$. The resonant QHA antenna is broadband device, hence its central frequency should be selected as fent $=162 \mathrm{MHz}$. Knowing central frequency value it makes simple to calculate wavelength for this central frequency, what is presented by formula:

$$
\lambda=\mathrm{c} / \mathrm{fcnt}=3 \times 1.8 / 162 \times 10^{6}=1.8[\mathrm{~m}]
$$

Using $\lambda=1.8[\mathrm{~m}]$ can be calculated necessary wire length Lele for open and shorted circuit of the QHA antenna. Other dimensions and parameters of the QHA array should be selected empirically during experiment to achieve desirable radiation pattern $[1,2,4,7]$.

Simulation and Testing

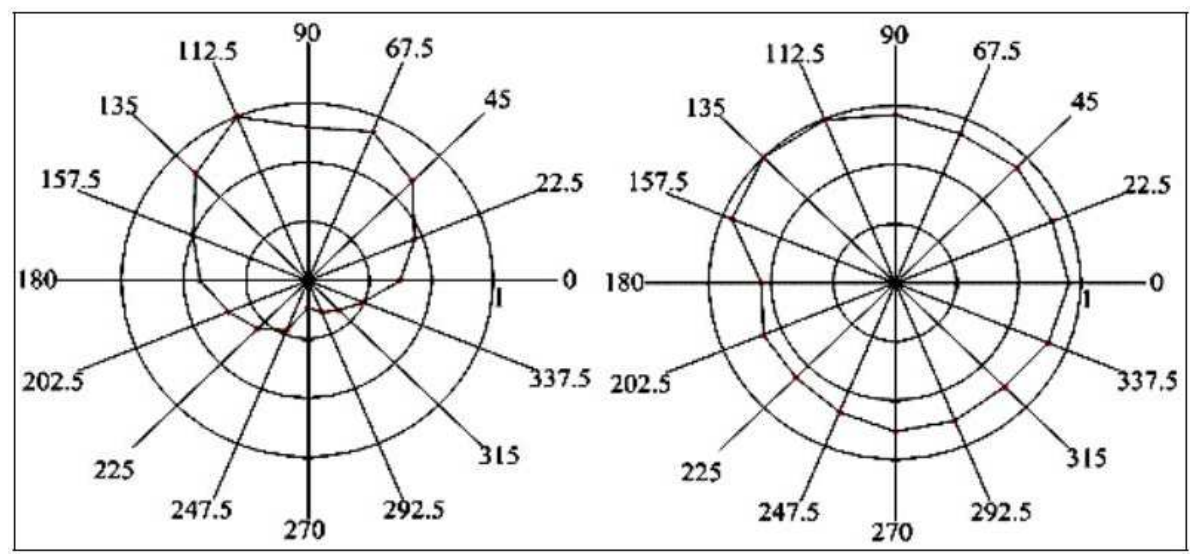

Figure 9. Radiation Pattern of QHA Antenna. 
Accordance to provided design and calculations for the QHA antenna in previous subsection, the QHA antenna was designed. The resonant QHA antenna has been implemented as "turn, quarter lambda" type. Two twisted wire frames are placed upon each other at right angles and connected to the antenna cable at the top (feeding provided at the top) with part of QHA-cylinder equals to approximately $0.4 \mathrm{~m}$. The photo of experimental sample of QHA antenna done at Durban University of Technology that can be used onboard ships simultaneous for both R-AIS and S-AIS is shown in Figure 8. For the measuring of radiation pattern of QHA was used special laboratory equipment which provides rotation of antenna with fixed steps of degrees in two plans what necessary to obtain measuring of radiation pattern antenna in two cross-sections.

The structure of fully equipped laboratory stands for measuring radiation pattern of the both QHA antennas can be done by connecting transmitting (TX) antenna to VHF generator for producing sinusoidal signal at $162 \mathrm{MHz}$ that corresponds to R-AIS central frequency. The receiving $(\mathrm{Rx})$ antenna is simple half-wave dipole connected to VHF detector that can show the level of receiving signal in relative units. The theoretical far-field region is 0.9 meter, which means that taken 5 meters of distance between Rx and Tx antenna for measuring of radiation pattern is sufficient for correct measurements. With help of the laboratory stand was also provided measurements of both vertical and horizontal crosssections of antenna radiation pattern. Measurements were provided with steps in 22.50 to achieve smooth curvatures. The measurement of the radiation patterns done in relative units is maximum magnitude in achieved radiation pattern of left or right unit shown in the Figure 8. In order to convert relative units into $\mathrm{dB}$, logarithmic equation should be used. In such a way, it will be necessary to take into account that measurements provided by the detector connected to antenna. Such detector provides measuring of antenna voltage, hence should be used logarithmic equation as follows:

$$
\mathrm{dB}=10 \log \left(\mathrm{U}^{2} / \mathrm{U} 0^{2}\right)
$$

where $\mathrm{U} 1$ = magnitude value of voltage given by detector; $\mathrm{U} 0=$ is threshold magnitude of voltage; and dB antenna gain. Therefore, in the left side of Figure 9 is shown radiation pattern with vertical cross-section and in the right side is shown radiation pattern with horizontal cross-section. Simple calculation with the help of the equation (8) is showing that the QHA antenna produces radiation patter with gain higher than $3 \mathrm{~dB}$ within confined angle of 100 and 1750, with the available values it is proved that reliable AIS is implemented.

\section{Conclusion}

Designed and assembled sample of the resonant QHA antenna clearly shows that the resonant QHA antenna can be used for both the radio-based (R-AIS) and satellite-based (SAIS). Such antenna should be improved by adding ground shield plane, which will prevent harmful back radiation being reflected from the sea surface. The next stage is to provide test of this antenna in real environment at sea. This antenna doesn't require any changes in the AIS equipment to be coupled. Also the resonant QHA antenna easily matches with input impedance of AIS transmitter, since resonant antenna has only resistive impedance.

\section{References}

[1] Fujimoto K. \& James JR. (1994) Mobile Antenna Systems Handbook", Artech House, Boston.

[2] Kraus J. D. and Others. (2002) Antennas for 11 Applications", McGraw-Hill, Columbus, $\mathrm{OH}$.

[3] (2014) IMO, Automati Identificatiom System”, Interanational Maritime Organisation, London.

[4] Ilcev D. S. (2011), "Global Mobile CNS", Manual, DUT, Durban.

[5] (2014) West Marine, "VHF Antenna", Watsonville, CA, [www.westmarine.com/WestAdvisor/Selecting-a-VHFAntenna].

[6] Gratton D. and Others (2011), "Marine Antenna Installations", NMEA, Severna Park, MD [www.nmea.org].

[7] Hollander R. W (1999), "Resonant Quadrifilar Helix Antenna" Working Group Satellites.

[8] Den Haag (2013) SRT Marine, "Marine Automatic Identification System", Midsomer Norton, Bath, UK, www.softwarerad.com.

[9] Eun Su Lee, Amit J. Mokashi, Sang Young Moon and Geun Sub Kimby Maturity of AIS and its Implications J. Mar. Sci. Eng. 2019, 7 (9), 287; https://doi.org/10.3390/jmse7090287 24 Aug 2019.

[10] A Dobrokovic, MV Lacob, JV Hillgesberg “ Maritime Pattern Extraction and route Reconstruction using AIS" International journal of Data science and Analytics Vol 5 Issue 3 PP 111 136.

[11] Chen Lihu, Liu Xianfeng, Yu Sunquan and Li Shiyou (2018) A Design of Space-borne AIS Scene Simulation Based on Density Distribution of Global Vessels IOP Conference Series: Materials Science and Engineering, Volume 449, Conf 1 PP 221-226.

[12] Lianbo Li, Wenyu Lu,, Jiawei Niu, Junpo Liu AIS Data-based Decision Model for Navigation Risk in Sea Areas Volume 71, Issue 3 May 2018, pp. 664-678 DOI: https://doi.org/10.1017/S0373463317000807.

[13] IV Kartika, NA Siwandari, Ari Vijayanti Application of Genetic Algorithm for Placement of AIS 2018 Electrical Power, Electronics, Communications, Controls and Informatics Seminar (EECCIS) DOI: 10.1109/EECCIS.2018.8692969.

[14] YJ Wu and S Zhang "Improved positioning method for Two base stations AIS" Sensors (Basel). 2018 Apr; 18 (4): 991. doi: $10.3390 / \mathrm{s} 18040991$.

[15] S. Huwai and S. Zhang A novel sparse representation algorithm for AIS real-time signals EURASIP Journal on Wireless Communications and Networking volume 2018, Article number: 223 (2018). 